\title{
Identification and Characterization of Circular RNAs in Longissimus Dorsi Muscle Tissue from Two Goat Breeds using RNA-Seq
}

\section{Jiyuan Shen}

Gansu Agricultural University

Huimin Zhen

Gansu Agricultural University

Lu Li

Gansu Agricultural University

Yuting Zhang

Gansu Agricultural University

Jiqing Wang ( $\nabla$ wangjq@gsau.edu.cn )

Gansu Agricultural University

Jiang Hu

Gansu Agricultural University

Xiu Liu

Gansu Agricultural University

\section{Shaobin Li}

Gansu Agricultural University

Zhiyun Hao

Gansu Agricultural University

Mingna Li

Gansu Agricultural University

\section{Zhidong Zhao}

Gansu Agricultural University

\section{Yuzhu Luo}

Gansu Agricultural University

\section{Research Article}

Keywords: circular RNAs, Longissimus dorsi muscle, goats, RNA-Seq

Posted Date: October 7th, 2021

DOI: https://doi.org/10.21203/rs.3.rs-944970/v1 
License: (c) (i) This work is licensed under a Creative Commons Attribution 4.0 International License. Read Full License

Version of Record: A version of this preprint was published at Molecular Genetics and Genomics on April 16th, 2022. See the published version at https://doi.org/10.1007/s00438-022-01887-1. 


\section{Abstract}

Background: Circular RNAs (circRNAs) are a class of non-coding RNA that play crucial roles in the development of skeletal muscle. However, little is known about the role of circRNAs in caprine skeletal muscle. In this study, the muscle fiber size and expression profiles of circRNAs were compared in Longissimus dorsi muscle of Liaoning cashmere (LC) goats and Ziwuling black (ZB) goats with significant phenotypic differences in meat production performance, using hematoxylin and eosin staining and RNA-Seq, respectively.

Results: The muscle fiber size in LC goats were larger than those in ZB goats $(P<0.05)$. A total of 10,875 circRNAs were identified and 214 of these were differentially expressed between the two caprine breeds. The authentication and expression levels of 20 circRNAs were confirmed using reverse transcriptasepolymerase chain reaction (RT-PCR) and DNA sequencing. The parent genes of differentially expressed circRNAs were mainly enriched in connective tissue development, Rap1, cGMP-PKG, cAMP and Ras signaling pathway. Some miRNAs reportedly associated with skeletal muscle development and intramuscular fat deposition would be targeted by several differentially expressed circRNAs and the most highly expressed circRNA (circ_001086).

Conclusion: These results provide an improved understanding of the functions of circRNAs in skeletal muscle development of goats.

\section{Background}

Circular RNAs (circRNAs) are a novel class of non-coding RNA and they are result from the covalently linage of 5' and 3' ends of linear RNA [1]. CircRNAs were first found in plant viroid in 1976 [2], which were initially regarded as the by-products originated from splicing errors of linear RNAs. However, it is now widely recognized that circRNAs generally exist in various eukaryotes. Due to the closed loop structure, circRNAs are not susceptible to affect by RNA exonucleases. It is therefore known that circRNAs are more stable and evolutionarily conserved than the linear mRNAs [3].

In recent years, more and more circRNAs were identified and their function are also gradually uncovered. CircRNAs mainly function as microRNA (miRNA) sponges. They thereby relieve the inhibition of target mRNAs by miRNAs, eventually resulting in an increase in expression level of target mRNAs [4-6]. For example, ciRS-7 can act as a miR-7 sponge to regulate the development of neurons and brain tumour in humans [7]. Additionally, exon-intron circRNAs predominantly localized in the nucleus can regulate the transcription of their parent genes in a cis-regulatory [8]. Finally, other functions of circRNAs have also been reported, including regulating alternative splicing [9], interacting with RNA-binding proteins [10], and even being translated into protein [11].

Skeletal muscle is the largest organ of animals and its growth and development directly determines meat production performance and meat quality in domestic animals. It is now found that many functional genes and non-coding RNAs are involved in the growth and development of skeletal muscle. 
However, compared with our knowledge of skeletal muscle mRNAs [12-14] and miRNAs [15-17], the reports on the roles of circRNAs in the skeletal muscle are very limited.

To date the studies of circRNAs in skeletal muscle tissues have mainly been focused on pigs [18-21], chickens [5,6,22]and cattle [23-25]. These studies reported the expression profiles and characterization of circRNAs in skeletal muscle either from different breeds, or from different development stages, and also analyzed the function of circRNAs more by investigating the functions of their parent genes using Gene Ontology (GO) and Kyoto Encyclopedia of Genes and Genomes (KEGG) analysis. For example, 13,377 circRNAs were identified in leg muscles of chickens between 11 embryo age, 16 embryo age and 1 day post hatch periods. Subsequently, one (circRBFOX2s) of the differentially expressed circRNAs identified was found to promote the proliferation of chicken myoblast by targeting miR-206 [5]. Additionally, a total of 655 circRNAs were found to be differentially expressed in muscle tissues between Shandong black cattle and Luxi cattle with significant differences in the diameter, length and weight of muscle fibers, and their parent genes were mainly involved in muscle fiber development process, and MAPK and mTOR signaling pathways [24].

However, there are only two reports about the activity of circRNAs in muscle tissues in goats. Ling et al. [26] identified 9,090 circRNAs in skeletal muscle of Anhui white goats across seven development stages, and the parent genes of the differentially expressed circRNAs were involved in the regulation of myoblast differentiation, skeletal muscle maturation and hypertrophy. In addition, a circRNA CDR1as has been found to activate the differentiation of caprine skeletal muscle satellite cells by relieving the inhibition of IGF1R targeted by miR-7 [27].

Liaoning cashmere (LC) goats and Ziwuling black (ZB) goats are famous local breeds in China and of economic importance for goat farmers. Compared with ZB goats, LC goats have higher carcass weight, content of intramuscular fat, and proportion of intramuscular collagen fibers, but poorer meat tenderness $(P<0.05)$ [28-29]. In this study, the circRNAs expression profiles of Longissimus dorsi muscle tissue were compared between LC and ZB goats using RNA-Seq. The GO enrichment and KEGG pathway were also analyzed for the parent genes of differentially expressed circRNAs between the two caprine breeds. The results will provide an improved understanding of the function of circRNAs in skeletal muscle growth and development processes in goats.

\section{Results}

\section{Comparison of the size of muscle fiber between LC and ZB goats}

Muscle fibers are the main structural components of skeletal muscle tissues, accounting for about $70 \%$ of the total weight. The dimeter and cross-sectional area are important morphology characteristics of muscle fibers and they play key roles in determining meat production performance of animals [33-36]. The comparison results of the dimeter and cross-sectional area of Longissimus dorsi muscle fiber 
between LC and ZB goats are shown in Figure 1. The dimeter and cross-sectional area of muscle fibers from LC goats were $38.64 \pm 2.19 \mu \mathrm{m}$ and $1948.34 \pm 135.76 \mu \mathrm{m}^{2}$, respectively, which was larger than ZB goats with a dimeter of $29.09 \pm 3.81 \mu \mathrm{m}$ and a cross-sectional area of $1117.72 \pm 210.10 \mu \mathrm{m}^{2}$ (Figure 1).

\section{The identification of circRNAs in the caprine Longissimus dorsi muscle tissues}

The raw reads obtained in the study from ten Longissimus dorsi muscle samples have been submitted to GenBank database with accession numbers SRR13008213-SRR13008222. The RNA-Seq reads and mapped results to the goat reference genome have also been described in our previous study [28]. Concisely, an average of $77,996,504$ and $82,000,854$ high quality clean reads could be mapped to Caprine

Genome Assembly ARS1 from LC and ZB goats, respectively. A total of 8,781 and 8,872 circRNAs were identified in Longissimus dorsi muscle tissues of LC and ZB goats, respectively, with 6,778 circRNAs being expressed at both breeds. Most notably, circ_001086 derived from LIM domain 7 (LMO7) was the most highly expressed circRNA in muscle tissues of both LC and ZB goats.

\section{Authentication of caprine circRNAs in the Longissimus dorsi muscle tissue}

A total of 20 circRNAs were selected to validate the presence of their specific head-to-tail junctions using RT-PCR and Sanger sequencing. The agarose gel electrophoresis results showed that all 20 circRNAs were expressed and they produced a band with an expected size (Figure 2A). Sanger sequencing further affirmed the presence of head-to-tail splice junctions and the size for these caprine circRNAs, which was consistent with those provided by RNA-seq (Figure 2B).

\section{Characterization of circRNAs identified in the caprine Longissimus dorsi muscle tissue}

Of the six types of circRNAs, annot_exons were the most common sequences with an average proportion of $78.03 \%$, followed by exon_intron, one_exon and antisense sequence types with an average proportion of $7.69 \%, 5.25 \%$, and $3.97 \%$, respectively. Intronic and intergenic sequences were the least common types with an average proportion of $3.14 \%$ and $1.91 \%$, respectively (Figure 3A). Meanwhile, for multiple circRNAs produced by a single gene, only 1-2 circRNAs had higher expression levels.

The circRNAs identified from Longissimus dorsi muscles were widely distributed across all the caprine chromosomes, with the exception of $Y$ chromosome. The most circRNAs were distributed on chromosomes 2 and 1, while the least circRNAs were distributed on chromosome 27 (Figure 3B). The length of the most circRNAs was less than $1 \mathrm{~kb}$ in length (Figure $3 \mathrm{C}$ ). 


\section{Identification and validation of differentially expressed circRNAs}

Of all the 10,875 circRNAs identified in caprine Longissimus dorsi muscle tissues, 214 circRNAs were found to be differentially expressed between LC and ZB goats, including 85 up-regulated circRNAs and 129 down-regulated circRNAs in Longissimus dorsi muscle of LC goats when compared to ZB goats (Supplementary File 1). The most up-regulated circRNA in LC goats was circ_008092 derived from signal transducerand activator of transcription 1 (STAT1) with a 19.4-fold increase in expression, while circ_003628 derived from myosin-4 (MYH4) was the most down-regulated circRNA in LC goats with a 55.6-fold decrease in expression. Most notably, of 214 differentially expressed circRNAs identified, 25 circRNAs were only expressed in LC goats, while 39 circRNAs were only expressed in ZB goats.

To validate the repeatability of RNA-seq data, 20 differentially expressed circRNAs that were used for the validation of the presence of circRNAs, were also subjected to RT-qPCR analysis. Because circ_006172 and circ_009217 were only expressed in LC and ZB goats, respectively, the $\log _{2}$ fold-change for LC goats relative to ZB goas was infinity for these two circRNAs. Their relative expression levels are therefore not presented in Figure 4. As shown in Figure 4, the expression levels of circ_009262, circ_003757, circ_008447, circ_002956, circ_007137, circ_001875, circ_006718, circ_008092, circ_008770, circ_009387, and circ_002473 in LC goats were higher than those in ZB goats. In contrast, compared to ZB goats, the expression levels of circ_005348, circ_004394, circ_003976, circ_008047, circ_010303, circ_007151, and circ_008117 were lower in LC goats. The results suggest that RT-qPCR results for these circRNAs in the Longissimus dorsi muscle were in accordance with those obtained from RNA-Seq (Figure 4), and that our RNA-Seq results are repeatable and reliable.

\section{Function annotation of the parent genes of differentially expressed circRNAs}

To further understand how these differentially expressed circRNAs regulate phenotypic differences in meat production performance and meat quality between the two goat breeds, GO enrichment and KEGG pathway analysis were performed for the parent genes of the differentially expressed circRNAs. The most significant GO term with the lowest $P$ value was connective tissue development $(P=6.43 \mathrm{E}-04)$, followed by syncytium formation by plasma membrane fusion $(P=0.001)$ and syncytium formation $(P=0.001)$. In addition, several important $\mathrm{GO}$ terms related to skeletal muscle hypertrophy were also found, including regulation of GTPase activity $(P=0.004)$, Rho GTPase binding $(P=0.005)$, Ras GTPase binding $(P=$ 0.006), small GTPase binding ( $P=0.011)$, and GTPase binding $(P=0.012)$ (Figure $5 \mathrm{~A})$.

Several significant KEGG pathways $(P<0.05)$ associated with the growth and development of skeletal muscle and the deposition of intramuscular fat were also found in this study, including Rap1 signaling pathway $(P=0.002)$, CGMP-PKG signaling pathway $(P=0.009)$, cAMP signaling pathway $(P=0.043)$, Ras signaling pathway $(P=0.045)$, and adipocytokine signaling pathway $(P=0.047)$ (Figure 5B). 


\section{Functional analysis of circRNAs as miRNA sponges}

For the 214 differentially expressed circRNAs identified, a total of 433 miRNAs were predicted to be target miRNAs. For clearly presenting the interaction effect of circRNAs and miRNAs, 7 circRNAs were further selected, including the most highly expressed circ_001086 in both caprine breeds, five up-regulated circRNAs (circ_002300, circ_006172, circ_008092, circ_001875, and circ_009387) and one down-regulated circRNA (circ_001709) in LC goats compared to ZB goats. There were 95 target miRNAs in total for these 7 circRNAs, ranging from 3 target miRNAs for circ_008092 to 27 target miRNAs for circ_001086. For each circRNA, the target miRNAs with the highest score were selected from 95 target miRNAs and then used to construct a circRNA-miRNA network (Figure 6). Some miRNAs that have been previously reported to be associated with the growth and development of skeletal muscle and the proliferation and differentiation of preadipocytes were found, including miR-140-3p, miR-129-5p, miR-424-5p, miR-27b-3p and miR-30a-3p [6,37-40].

\section{Discussion}

In this study, the diameter and cross-sectional area of Longissimus dorsi muscle fiber from LC goats were larger than those from ZB goats. It has been reported that the diameter or cross-sectional area reflects the size of muscle fiber, which directly determines skeletal muscle mass during postnatal period of animals $[41,42]$. This may partly explain why in our findings LC goats had higher carcass weight than ZB goats. Choi and Oh [36] also found that pigs with greater cross-sectional area of muscle fibers had higher carcass weight $(P<0.001)$. Besides carcass weight, cross-sectional area of muscle fiber has been reported to positively correlated with intramuscular fat content $(r=0.68)$ of pork [34], and muscle fiber diameter was also positively correlated with shear force value $(r=0.63)$ and loin eye area $(r=0.56)$ of beef [33]. These studies further supported our observation that meat from LC goats with higher diameter and cross-sectional area of Longissimus dorsi muscle fiber had higher muscle shear force value, intramuscular fat content and loin eye area than meat from ZB goats.

In our study, an average of 10,875 circRNAs were identified in Longissimus dorsi muscle from goats. The number of circRNAs identified in the study was higher than what was investigated by Ling et al. [26], who described 9,090 circRNAs in caprine Longissimus dorsi muscle tissues of Anhui white goats. Additionally, 14,640 and 6,988 circRNAs were found in muscle tissues from cattle [24] and pigs [20], respectively. This likely revealed species-specific expression pattern of circRNAs.

Our observation that most of circRNAs identified were the type of annot_exons, was in accordance with the findings in muscle tissues in Anhui white goats [26], pigs [43], cattle [23], and chicken [5]. Other types of circRNAs that have been identified in Longissimus dorsi muscle of Anhui white goats [26], chicken [5] and pigs [21], were also found in the study. For example, intronic and intergenic circRNAs accounted for $5.10 \%$ and $2.49 \%$ of all circRNAs detected in Longissimus dorsi muscle tissues from Anhui white goats [26]. The proportion of exon_intron and antisense circRNAs were $7.23 \%$ and $1.41 \%$ in all circRNAs found in muscle tissues of pigs [21]. Most of circRNAs identified in this study were less than $1 \mathrm{~kb}$ in 
length, which was also consistent with the length distribution of circRNAs reported in skeletal muscle of cattle [23] and pigs [44]. It is noteworthy that of multiple circRNAs produced by a single gene, there were only 1-2 circRNAs with a higher expression level. For example, caprine $L M O 7$ produced a total of 13 circRNAs in the study, whereas only circ_001086 and circ_008196 expressed at higher levels with RPM values of $>1,000$. This phenomenon was also observed in bovine muscle circRNAs [23]. Caprine chromosomes 2 and 1 produced the most circRNAs, while chromosome 27 produced the least circRNAs in this study. It is perhaps unsurprising as caprine chromosomes 1 and 2 are the largest in size, while chromosome 27 is relatively small in the goat genome. Ling et al. [26] also found similar chromosome distribution of circRNAs in skeletal muscle tissues of Anhui white goats. Studies in cattle, chicken and pigs also confirmed that the numbers of circRNAs found was proportional to chromosome size [22, 23, 44].

It was noteworthy that the most highly expressed circRNA in both LC and ZB goats was circ_001086 derived from $\angle M 07$. $\angle M O 7$ was essential to skeletal muscle development as it maintains proper myoblast differentiation [45]. On contrary, knockdown of LMO7 inhibited myogenesis by preventing myotube formation and decreasing the number of myoblasts in chicken [46]. Additionally, in bovine Longissimus dorsimuscle tissue, circLMO7 produced from $\angle M O 7$ was the most down-regulated circRNA at adult stage compared to embryonic period, and the circRNA also promoted the proliferation of myoblast, but inhibited the differentiation and apoptosis of myoblasts by sponging miR-378a-3p [23]. These suggest that circ_001086 play key roles for skeletal muscle development in both LC and ZB goats and it is worthy of further investigation.

Compared to ZB goats, the most up-regulated and down-regulated circRNAs in LC goats were circ_008092 and circ_003628, respectively, which originated from STAT1 and MYH4, respectively. STAT1 has been reported to play a positive role in myoblast proliferation and hypertrophy of skeletal muscles [47-48]. In addition, our circRNA-miRNA interaction network showed that circ_008092 would act as miR-330-5p sponge (Figure 6). The miR-330-5p negatively regulated ovine preadipocyte differentiation [15]. It was therefore inferred that the up-regulated expression of circ_008092 in Longissimus dorsi muscle of LC goats may be responsible for its higher carcass weight and intramuscular fat content. Our target miRNAs prediction also found that circ_003628 would sponge either miR-217-5p positively related to skeletal muscle cell proliferation [49], or miRNAs positively related to skeletal muscle cell differentiation, including miR-1 [50], miR-26a [51], miR-27b [52] and miR-148a-3p [53]. These suggests that the lower expression of circ_003628 in LC goats may contribute to its higher meat production performance by less inhibition of the expression levels of these important miRNAs.

Connective tissue development was the most significant GO term enriched for the parent genes of some differentially expressed circRNAs. These circRNAs included LOC102187872 circRNA (circ_002339), SOX6 circRNA (circ_006718), ZBTB16 circRNA (circ_008022), ADAMTS12 circRNA (circ_004981), and CREB5 circRNA (circ_000980). The parent genes of these circRNAs have been found to be associated with the synthesis of collagen fibers, which mainly form intramuscular connective tissue. For example, LOC102187872 encodes collagen alpha-1 (V) chain, and the protein SOX6 actived the expression of the 
gene encoding collagen type 2 by combing with a 48 bp enhancer [54]. Taken together, the differentially expression of these circRNAs may partly explain the difference in intramuscular collagen fiber content between LC and ZB goats.

Interestingly, when the same samples as the study were used to compare the difference in transcriptome profile of Longissimus dorsi muscle tissues between LC and ZB goats, LOC102187872 was found to be an up-regulated gene in LC goats [28]. These suggests that circ_002339 had the opposite expression tendency with its parent gene LOC102187872. Genome wide analysis results showed that the expression level of $38.7 \%$ circRNAs identified in embryonic muscle tissue of pigs had a strong correlation $(|\gamma\rangle 0.9)$ with their parent genes. Of these circRNAs, the expression level of $9.0 \%$ circRNAs were negatively correlated with their parent genes [18]. Unlike circ_002339, the expression tendency of circ_005286 and circ_007643 between LC and ZB goats was consistent with their parent genes LOC102191280 and LOC102190983, respectively, namely these circRNAs and their parent genes were all down-regulated in Longissimus dorsi muscle from LC goats compared to ZB goats. Taken together these suggest that there is a complex relationship in expression between circRNA and its parent gene.

The skeletal muscle growth during postnatal period mainly depends on the hypertrophy of muscle fiber. The mTOR pathway was one of the most important factors promoting skeletal muscle hypertrophy by facilitating muscle protein synthesis [41]. It has been reported that the effect of mTOR pathway on muscle fiber depends on various GTPases $[55,56]$. In the study, some differentially expressed circRNAs were enriched in GO terms related to GTPases, which included regulation of GTPase activity, GTPase binding, small GTPase binding, Rho GTPase binding, and Ras GTPase binding. The circ_003976 and circ_007919 are one of these differentially expressed circRNAs. FNIP1 is the parent gene of circ_003976, and involved in the accumulation of crucial muscle proteins such as myosin heavy chain and troponins [57]. CYFIP1 producing circ_007919 promoted the remodeling of actin, which is one of the most important muscle proteins [58]. It was therefore inferred that differentially expression of these circRNAs in Longissimus dorsi muscle tissues between LC and ZB goats may be responsible for significant phenotypic differences in carcass weight originated from muscle fiber hypertrophy.

Nine differentially expressed circRNAs attracted our attention as their parent genes were significantly enriched in CAMP signaling pathway, Ras signaling pathway, and cGMP-PKG signaling pathway, which were closely associated with growth and development of skeletal muscle and adipose tissue. These consisted of MAPK1 circRNA (circ_001875), AKT3 circRNA (circ_001709), MET circRNA (circ_009387), MEF2A circRNA (circ_006172), NFKB1 circRNA (circ_002300), PLCE1 circRNA (circ_008117), AFDN circRNA (circ_007151), PLCB4 circRNA (circ_001835), and CREB5 circRNA (circ_000980). The cAMP signaling pathway is crucial for skeletal muscle hypertrophy [59] and also associated with adipocyte differentiation and lipolysis [60]. Ras signaling was involved in inhibition of myoblast differentiation and skeletal myogenesis [61,62], and regulation of adipocyte differentiation during brown adipogenesis [63]. The cGMP-PKG signaling mainly regulated skeletal muscle contraction [64] and adipocyte differentiation and lipolysis [65], and the pathway was also enriched for the parent genes of differentially 
expressed circRNAs in Longissimus dorsi muscle of Anhui white goats between different development stages [26].

As might be expected, the parent genes of the nine differentially expressed circRNAs described above were related with muscle hypertrophy and atrophy. For example, the proteins MAPK1 and AKT3 promoted the hypertrophy of postnatal skeletal muscle [66], and also participated in terminal differentiation and proliferation of myoblast [67-68]. The protein MET has been found to promote muscle hypertrophy by preventing apoptosis of myogenic progenitors [69-70]. The significant role of MEF2A has well been established in controlling embryonic myogenesis, adult skeletal muscle growth, hypertrophy and regeneration [71]. The knockout of NFKB1 inhibited the unloading-induced muscle atrophy by increasing cross-sectional areas of muscle fiber [72]. Meanwhile, the regulation effects of parent genes MAPK1, AKT3 and MEF2A on adipogenesis have also been described [73-75]. These indicate that the parent genes of these differentially expressed circRNAs detected contributed to the differences in carcass weight and content of intramuscular fat between LC and ZB goats.

Other differentially expressed circRNAs of interest, which their parent genes are crucial for skeletal muscle growth and development, included WWP1 circRNA (circ_008374), RNF13 circRNA (circ_007619), STAU2 circRNA (circ_00404), and STAU2 circRNA (circ_000993). WWP1 has been reported to regulate skeletal muscle hypertrophy and atrophy [76], while RNF13 and STAU2 were involved in the regulation of myoblast proliferation and differentiation [77-78].

The circRNAs can function as miRNAs sponge to positively regulate the expression levels of the target genes. In this context, the roles of circRNAs in various cell activities can be reflected by the functions of their target miRNAs. In the study, some predicted target miRNAs have previously been reported to be associated with skeletal muscle development and intramuscular fat deposition. For example, as the most highly expressed circRNA in both two caprine breeds, circ_001086 would target multiple miRNAs, including miR-103-3p, miR-129-5p, miR-140-3p, miR-330-5p, miR-335-5p, miR-423-5p, and miR-532-3p (Figure 6). Of these target miRNAs, miR-140-3p, miR-423-5p, and miR-532-3p inhibited skeletal muscle myogenesis by suppressing myoblast fusion, skeletal muscle satellite cells differentiation, and myoblast proliferation $[37,16,17]$. Additionally, miR-129-5p and miR-330-5p has been reported to inhibit preadipocyte proliferation [38] and differentiation [15] by targeting G3BP1 and $B C A T 2$, respectively.

The miR-424-5p and miR-15b-5p would be targeted by circ_001875 (Figure 6), which was up-regulated in Longissimus dorsi muscle of LC goats with higher carcass weight. Previous studies found that miR-424$5 p$ decreased human skeletal muscle mass by reducing protein synthesis [39], and miR-15b-5p suppress myoblast proliferation and differentiation by regulating IGF1-PI3K/AKT pathway [79]. It was therefore inferred that the higher expression level of circ_001875 may contribute to higher muscle mass of LC goats by suppressing the negative effect of miR-424-5p and miR-15b-5p on skeletal development. Besides these miRNAs described above, miR-27b-3p that would be targetd by circ_002300 and circ_006172, and miR-30a-3p that would be targeted by circ_006172, also played important roles in 
proliferation and differentiation of myoblast in chicken $[6,40]$. These results indicate that these differentially expressed circRNAs identified in the study may play key miRNAs sponge roles in regulating the differences in meat production performance between LC and ZB goats.

\section{Conclusion}

A total of 214 circRNAs were differentially expressed between the two goat breeds with significant phenotype difference in meat production performance. The parent genes and target miRNAs of differentially expressed circRNAs were associated with muscle development and intramuscular fat deposition. This study provides an improved understanding of the roles of circRNAs in skeletal muscle development of goats. Moreover, this study lays the foundation for further research into the function of individual circRNAs in the development of muscle and adipose tissues.

\section{Methods}

\section{Ethics statement}

All experimental procedures were approved by Faculty of Animal Science and Technology, Gansu Agricultural University, Lanzhou, China. The investigation for experimental animals was also coincide with the rules for animal care and use published by the Ministry of Science and Technology of the People's Republic of China (Approval number 2006-398).

\section{Experimental animals and sampling}

The same Longissimus dorsi muscle samples as described by Shen et al. [28] were used in this study. Briefly, five nine-month-old LC rams and five nine-month-old ZB rams were selected from the Yongfeng Goat Breeding Company (Huan County, China) and then slaughtered. These goats were raised under the same feeding and management conditions. The carcass weight, muscle components and meat quality of these goats investigated in the study are presented in Table 1 [28-29].

The Longissimus dorsi muscle samples from the area between 12th and 13th ribs on the left carcass were collected and then used for RNA isolation and hematoxylin and eosin staining. The samples for RNA isolation were frozen in liquid nitrogen, whereas the samples for hematoxylin and eosin staining was fixed with $4 \%$ paraformaldehyde.

Table 1

The carcass weight, muscle components and meat quality of the two goat breeds 


\begin{tabular}{llll} 
& & \multicolumn{2}{l}{ goats $(\mathrm{n}=$} \\
& & $5)$ & \\
\hline Carcass weight $(\mathrm{kg})$ & $14.10 \pm 1.17$ & $7.45 \pm 1.28$ & $\begin{array}{l}2.600 \mathrm{E}- \\
05\end{array}$ \\
\hline Lion-eye area $\left(\mathrm{cm}^{2}\right)$ & $13.44 \pm 2.26$ & $5.86 \pm 1$. & $\begin{array}{l}4.836 \mathrm{E}- \\
04\end{array}$ \\
\hline Muscle shear force value $(\mathrm{N})$ & & 97 & 0.027 \\
\hline $\begin{array}{l}\text { The proportion of intramuscular collagen } \\
\text { fibers (\%) }\end{array}$ & $17.78 \pm 1.21$ & $18.11 \pm$ & 1.27 \\
\hline Intramuscular fat content (\%) & & $12.31 \pm$ & 0.017 \\
\hline
\end{tabular}

\section{Hematoxylin and eosin staining of Longissimus dorsi muscle}

The Longissimus dorsi muscle samples fixed with $4 \%$ paraformaldehyde were treated using graded ethanol $(75,85,95$, and $100 \%)$ to remove moisture. Subsequently, the dehydrated specimens were embedded in paraffin and then cut into about $5 \mu \mathrm{m}$ of thickness using Rotary cutting machine (Leica, Wetzlar, Germany). The paraffin sections were used for hematoxylin and eosin staining as suggested by Cao et al. [30].

Micrographs (400x) of hematoxylin and eosin staining from three different fields of view for each sample were taken by BA200 Digital microscope (MOTIC, Xiamen, China). The diameter and cross-sectional area of muscle fibers were then measured using Motic Images Advanced v3.2. The difference in these measurements between LC and ZB goats was analyzed by SPSS v24.0.

\section{RNA samples preparation and sequencing}

Total RNA was isolated from ten caprine Longissimus dorsi muscle samples using Trizol reagent (Invitrogen, Carlsbad, CA, United States). The integrity and concentration of these RNA samples were measured using Agilent 2100 Bioanalyzer (Agilent, CA, United States) and Nanodrop 2000 (Thermo Scientific, MA, United States), respectively. High quality RNA samples were screened with a parameter of RNA integrity number $>7$ being fitted as the threshold. The ribosomal RNA (rRNA) was removed from these high quality RNA samples using a Ribo-Zero Gold rRNA Removal Kit (Illumina, CA, United States). The remaining RNA was fragmented into 200-300 bp in length and then used for constructing cDNA libraries using a NEBNext Ultra RNA Library Prep Kit for Illumina (New England Biolabs, MA, United 
States). The cDNA libraries were finally paired-end sequenced by a HiSeq ${ }^{\mathrm{TM}} 4000$ sequencer (Illumina, CA, United States).

\section{Analysis of RNA-seq data}

The clean reads were obtained by removing the reads with quality scores $<$ Q20, reads containing sequencing adapters and reads with $>10 \%$ unknown nucleotides from raw reads produced from sequencer, using fastp v0.18.0. These clean reads were then mapped to Caprine Genome Assembly ARS1 (ftp://ftp.ncbi.nlm.nih.gov/ genomes/all/GCF/001/704/415/GCF_001704415.1_ARS1) using HISAT2 v2.1.0. For the reads that were unmapped against Caprine Genome Assembly ARS1, 20-mers from both ends were defined as anchors reads. The anchor reads were mapped to the reference genome again using bowtie2 v2.2.8 and circRNAs were finally identified using software Find_circ [31]. The identified circRNAs were characterized by counting their chromosomal distribution, type and length. The expression level of each annotated circRNA was normalized by calculating the Reads Per Million mapped reads (RPM). The DESeq v2.0 was used to screen differentially expressed circRNAs in Longissimus dorsi muscle tissues between LC and ZB goats, with fold change $>2.0$ and $P$-value $<0.05$.

\section{Validation of the authenticity of circRNAs using reverse transcriptase-PCR and DNA sequencing}

Based on the characteristic that circRNA has a unique head-to-tail junction, its authenticity was validated using reverse transcriptase-PCR (RT-PCR) and DNA sequencing. Briefly, 20 differentially expressed circRNAs between the two caprine breeds were selected. These circRNAs were performed RTPCR analysis and then sequenced using RNA samples isolated originally for RNA-Seq. The information of divergent primers for these circRNAs are shown in Table 2. To confirm the authenticity of these circRNAs, the sequences from Sanger sequencing were aligned to the goat reference genome and RNASeq data to validate the location of the junction sites of these circRNAs.

Table 2

The information of primers used for RT-PCR and RT-qPCR 


\begin{tabular}{|c|c|c|}
\hline CircRNA/Gene & Forward $\left(5^{\prime} \rightarrow 3^{\prime}\right)$ & Reverse $\left(5^{\prime} \rightarrow 3^{\prime}\right)$ \\
\hline circ_009262 & АCCAACTGCTTTCCGAAGTG & GCTTCATTAAAGCCCACCAA \\
\hline circ_003757 & CAGATCCGAATGCAACTA & GCAACATCAATGCTACGC \\
\hline circ_008447 & TGCAGACGACGATAACTTGG & TCTTTATCACACAGAACTTGTTC \\
\hline circ_002956 & CATGGTTCTTCTGGTTTTGGA & TAGGAAAGGAGTTCCCAGCA \\
\hline circ_007137 & CCAGGAAGTGAAGGAAGCAG & TGCCСАCTGTCCAAАCTTCT \\
\hline circ_001875 & САСCTGCGATCTCAAGCTCT & GCCCGAATAATGTCGTTGAT \\
\hline circ_006718 & AAGGGCCTAAGTGACCGTTT & TGACCCTGAACCTGCTCAAT \\
\hline circ_008092 & TCAAGACCCTAGAAGATTTGCA & CATGAAAACGGATGGTGGCA \\
\hline circ_008770 & СCССTAACCGAGCTACTTCA & TCTGCGCTTTTTAACCCAAG \\
\hline circ_009387 & CGAATGTTATACCCCAGCCC & GGAAATGTTCACAGCCCAGG \\
\hline circ_006172 & GATCTTCGAGTTGTCATCCCC & AGCTTCTAGTTTGAGGGCTACA \\
\hline circ_005348 & GAGAGTTTCCGGGAGAGGAC & СTCCAGGTAGCTGTGGAAGG \\
\hline circ_004394 & GCTCCСАTCATCCTTGAAAA & GCTCCCAGATTAATGCTCCA \\
\hline circ_003976 & GAAAATGAGCCGGAGATCAG & GAGCGTCGCTACAGAGCTTC \\
\hline circ_002473 & AACAGTTGGCTTTTGACCCA & СCCTCGATCACTGAACAGGA \\
\hline circ_008047 & GGTTGCCACACACCTTCAAT & AGATCTATGGGCTTCGCAGG \\
\hline circ_010303 & CAACAACGACСССТTCTCAG & GTCCCCAATGATGTTGAGCG \\
\hline circ_007151 & TAAGAAGCACACGGATGGGA & TAGTGGACAGCAGGATCGTC \\
\hline circ_008117 & ССААСТССССАССТGСТААТ & AGGGGATCTTGGTTGTCAGG \\
\hline circ_009217 & AGATCTCACAGAACAGTAGCCA & СССАСТTGСТTАССТGТССТ \\
\hline GAPDH & ACACTGAGGACCAGGTTGTG & GACAAAGTGGTCGTTGAGGG \\
\hline$\beta$-actin & АGССTTCСTTCCTGGGCATGGA & GGACAGCACCGTGTTGGCGTAA \\
\hline
\end{tabular}

\section{Validation of the reliability of RNA-Seq results using RT- quantitative PCR}

The same 20 differentially expressed circRNAs as those used for RT-PCR, were selected to confirm the reliability of RNA-Seq results using RT-quantitative PCR (RT-qPCR). The ten RNA samples that were used for RNA-Seq were also used to produce cDNA using a RT-PCR kit (Takara, Dalian, China). The RT-qPCR was performed in triplicate using a $2 \times$ ChamQ SYBR qPCR Master Mix (Vazyme, Nanjing, China) on an 
Applied Biosystems QuantStudio ${ }^{\circledR} 6$ Flex (Thermo Lifetech, MA, USA). Caprine GAPDH and $\beta$-actin were used as internal control for normalizing the expression levels of these circRNAs as suggested by Ling et al. [26] and Wang et al. [20]. A $2^{-\Delta \Delta C t}$ method was finally used to calculate the relative expression levels.

\section{Function enrichment analysis of the parent genes of differentially expressed circRNAs and target miRNAs prediction of circRNAs}

The main function of the parent genes of the differentially expressed circRNAs was analyzed using GO database (http://www.geneontology.org/) and KEGG database [32]. Meanwhile, six differentially expressed circRNAs and the most highly expressed circRNA (circ_001086) in both caprine breeds were used to predict their target miRNAs using Mireap v0.2, Miranda v3.3a and TargetScan v7.0. The predicted results from the three kinds of software were intersected. An interaction network between these circRNAs and their target miRNAs was constructed using StarBase v3.0 and then drawn using Cytoscape v3.5.1.

\section{Abbreviations}

circRNAs: Circular RNAs

miRNA: microRNA

LC: Liaoning cashmere

ZB: Ziwuling black

RT-PCR: reverse transcriptase-polymerase chain reaction

STAT1: signal transducerand activator of transcription 1

MYH4: myosin-4

LMO7: LIM domain 7

\section{Declarations}

\section{Acknowledgements}

Not applicable.

\section{Author contributions}


JS, JW, YL conceived and designed the experiments. JS, HZ, LL, YZ performed the experiments. JS analyzed the data. JW, YL, JH, XL, SL, ZH, ML, ZZ contributed reagents, materials and tools and collected the samples. JS and JW wrote the manuscript and revised the manuscript. All authors read and approved the final manuscript.

\section{Funding}

This research was funded by the fund for Basic Research Creative Groups of Gansu Province (18JR3RA190), the Fuxi Young Talents Fund of Gansu Agricultural University (Gaufx-02Y02) and the Projects of Gansu Agricultural University (GSAU-ZL-2015-033).

\section{Availability of data and materials}

All raw transcriptome data in this study have been deposited to GenBank database with accession numbers SRR13008213-SRR13008222.

\section{Ethics approval and consent to participate}

Ethical approval by the Ethics Committee of Gansu Agricultural University, was obtained (GAU-LC-202027).

\section{Consent for publication}

Not applicable.

\section{Competing interests}

The authors declare no competing interests.

\section{References}

1. Lasda E, Parker R, Circular RNAs: diversity of form and function. RNA. 2014; 20: 1829-1842.

2. Sanger HL, Klotz G, Riesner D, Gross HJ, Kleinschmidt AK. Viroids are single-stranded covalently closed circular RNA molecules existing as highly base-paired rod-like structures. P Natl Acad Sci USA. 1976; 73: 3852-3856.

3. Jeck WR, Sharpless NE. Detecting and characterizing circular RNAs. Nat Biotechnol. 2014; 32: 45361. 
4. Hansen TB, Jensen TI, Clausen BH, Bramsen JB, Finsen B, Damgaard CK, Kjems J. Natural RNA circles function as efficient microRNA sponges. Nature. 2013; 495: 384-388.

5. Ouyang H, Chen X, Wang Z, Yu J, Jia X, Li Z, Luo W, Abdalla BA, Jebessa E, Nie Q, Zhang X. Circular RNAs are abundant and dynamically expressed during embryonic muscle development in chickens. DNA Res. 2018; 25: 71-86.

6. Chen B, Yu J, Guo L, Byers M, Wang Z, Chen X, Xu H, Nie Q. Circular RNA circhipk3 promotes the proliferation and differentiation of chicken myoblast cells by sponging miR-30a-3p. Cells. 2019; 8: 177.

7. Hansen TB, Jensen TI, Clausen BH, Bramsen JB, Finsen B, Damgaard C, Kjems KJ. Natural RNA circles function as efficient microRNA sponges. Nature. 2013; 495: 384-388.

8. Li Z, Huang C, Bao C, Chen L, Lin M, Wang X, Zhong G, Yu B, Hu W, Dai L, et al. Exon-intron circular RNAs regulate transcription in the nucleus. Nat Struct Mol Biol. 2015; 22: 256-264.

9. Ashwal-Fluss R, Meyer M, Pamudurti NR, Ivanov A, Bartok O, Hanan M, Evantal N, Memczak S, Rajewsky N, Kadener S. CircRNA biogenesis competes with pre-mRNA splicing. Mol Cell. 2014; 56: 55-66.

10. Hentze MW, Preiss T. Circular RNAs: splicing's enigma variations. EMBO J. 2014; 32: 923-925.

11. Wang Y, Wang Z. Efficient backsplicing produces translatable circular mRNAs. RNA. 2015; 21: 172179.

12. Hernández-Hernández JM, García-González EG, Brun CE, Rudnicki MA. The myogenic regulatory factors, determinants of muscle development, cell identity and regeneration. Semin Cell Dev Biol. 2017; 72: 10-18.

13. Taylor MV, Hughes SM. Mef2 and the skeletal muscle differentiation program. Semin. Cell Dev Biol. 2017; 72: 33-44.

14. Zammit PS. Function of the myogenic regulatory factors Myf5, MyoD, Myogenin and MRF4 in skeletal muscle, satellite cells and regenerative myogenesis. Semin Cell Dev Biol. 2017; 72: $19-32$.

15. Tao S, Yan X, Qiao L, Li B, Liu W. MiR-330-5p negatively regulates ovine preadipocyte differentiation by targeting branched-chain aminotransferase 2. Anim Sci J. 2018; 89: 858-867.

16. Cheng X, Li L, Shi G, Chen L, Li C. MEG3 promotes differentiation of porcine satellite cells by sponging miR-423-5p to relieve inhibiting effect on SRF. Cells. 2020; 9: 449.

17. Cai R, Zhang Q, Wang Y, Yong W, Pang W. Lnc-RNA interacts with microRNA-532-3p and IGF2BP2 to inhibit skeletal muscle myogenesis. J Biol Chem. 2021; 296: 100376.

18. Hong L, Gu T, He Y, Zhou C, Hu Q, Wang X, Zheng E, Huang S, Xu Z, Yang J, et al. Genome-Wide analysis of circular RNAs mediated ceRNA regulation in porcine embryonic muscle development. Front Cell Dev Biol. 2019; 7: 289.

19. Shen L, Gan M, Tang Q, Tang G, Jiang Y, Li M, Chen L, Bai L, Shuai S, Wang J, et al. Comprehensive analysis of IncRNAs and circRNAs reveals the metabolic specialization in oxidative and glycolytic skeletal muscles. Int J Mol Sci. 2019; 20: 2855. 
20. Wang J, Ren Q, Hua L, Chen J, Zhang J, Bai H, Li H, Xu B, Shi Z, Cao H, et al. Comprehensive analysis of differentially expressed mRNA, IncRNA and circRNA and their ceRNA networks in the Longissimus dorsi muscle of two different pig breeds. Int J Mol Sci. 2019; 20: 1107.

21. Li B, Yin D, Li P, Zhang Z, Zhang X, Li H, Li R, Hou L, Liu H, Wu W. Profiling and functional analysis of circular RNAs in porcine fast and slow muscles. Front Cell Dev Biol. 2020; 8: 322.

22. Shen X, Liu Z, Cao X, He H, Han S, Chen Y, Cui C, Zhao J, Li D, Wang Y. et al. Circular RNA profiling identified an abundant circular RNA circTMTC1 that inhibits chicken skeletal muscle satellite cell differentiation by sponging miR-128-3p. Int J Biol Sci. 2019; 15: 2265-2281.

23. Wei X, Li H, Yang J, Hao D, Dong D, Huang Y, Lan X, Plath M, Lei C, Lin F, et al. Circular RNA profiling reveals an abundant circLMO7 that regulates myoblasts differentiation and survival by sponging miR-378a-3p. Cell Death Dis. 2017; 8: e3153.

24. Liu R, Liu X, Bai X, Xiao C, Dong Y. Identification and characterization of circRNA in Longissimus dorsi of different breeds of cattle. Front Genet. 2020; 11: 565085.

25. Yan XM, Zhang Z, Meng Y, Li HB, Gao L, Luo D, Jiang H, Gao Y, Yuan B, Zhang JB. Genome-wide identification and analysis of circular RNAs differentially expressed in the longissimus dorsi between Kazakh cattle and Xinjiang brown cattle. Peer J. 2020; 8: e8646.

26. Ling Y, Zheng Q, Zhu L, Xu L, Sui M, Zhang Y, Liu Y, Fang F, Chu M, Ma Y, et al. Trend analysis of the role of circular RNA in goat skeletal muscle development. BMC Genomics. 2020; 21: 220.

27. Li L, Chen Y, Nie L, Ding X, Zhang X, Zhao W, Xu X, Kyei B, Dai D, Zhan S, et al. MyoD-induced circular RNA CDR1 as promotes myogenic differentiation of skeletal muscle satellite cells. BBA-Gene Regul Mech. 2019; 1862: 807-821.

28. Shen J, Hao Z, Wang J, Hu J, Liu X, Li S, Ke N, Song Y, Lu Y, Hu L, et al. Comparative transcriptome profile analysis of Longissimus dorsi muscle tissues from two goat breeds with different meat production performance using RNA-Seq. Front Genet. 2021; 11: 619399.

29. Wang J, Shen J, Liu X, Li S, Luo Y, Zhao M, Hao Z, Ke N, Song Y, Qiao L. Comparative analysis of meat production traits, meat quality, and muscle nutrient and fatty acid contents between Ziwuling black goats and Liaoning cashmere goats. Acta Pratacult Sin. 2021; 30: 166-177.

30. Cao T, Shi L, Zhang L, Zhou H, Xun W, Hou G. Comparative study on fetal muscle fiber of Wuzhishan pig and Changbai pig during 65d gestation. J Anim Ecol. 2014; 35: 37-40.

31. Memczak S, Jens M, Elefsinioti A, Torti F, Krueger J, Rybak A, Maier L, Mackowiak SD, Gregersen LH, Munschauer $\mathrm{M}$, et al. Circular RNAs are a large class of animal RNAs with regulatory potency. Nature. 2013; 495: 333-338.

32. Kanehisa M, Araki M, Goto S, Hattori M, Hirakawa M, Itoh M, Katayama T, Kawashima S, Okuda S, Tokimatsu T, et al. KEGG for linking genomes to life and the environment. Nucleic Acids Res. 2008; 36: D480-D484.

33. Tuma HJ, Venable JH, Wuthier PR, Henrickson RL. Relationship of fiber diameter to tenderness and meatiness as influenced by bovine age. J Anim Sci. 1962; 33-36. 
34. Larzul C, Lefaucheur L, Ecolan P, GoguéJ, Talmant A, Sellier P, Le Roy P, Monin G. Phenotypic and genetic parameters for longissimus muscle fiber characteristics in relation to growth, carcass, and meat quality traits in large white pigs. J Anim Sci. 1997; 75: 3126-3137.

35. Rehfeldt C, Fiedler I, Dietl G, Ender K. Myogenesis and postnatal skeletal muscle cell growth as influenced by selection. Livest Prod Sci. 2000; 66: 177-188.

36. Choi YM, Oh HK. Carcass performance, muscle fiber, meat quality, and sensory quality characteristics of crossbred pigs with different live weights. Korean J Food Sci An. 2016; 36: 389-396.

37. Luo W, Li E, Nie Q, Zhang X. Myomaker, regulated by MyoD, MyoG and mir-140-3p, promotes chicken myoblast fusion. Int J Mol Sci. 2015; 16: 26186-26201.

38. Lv S, Ma M, Sun Y, Wang X, Qimuge N, Qin J, Pang W. MicroRNA-129-5p inhibits 3T3-L1 preadipocyte proliferation by targeting G3BP1. Anim Cells Syst. 2017; 21, 269-277.

39. Connolly M, Paul R, Farre-Garros R, Natanek SA, Bloch S, Lee J, Lorenzo JP, Patel H, Cooper C, Sayer $A A$, et al. miR-424-5p reduces ribosomal RNA and protein synthesis in muscle wasting. $J$ cachexia sarcopeni. 2018; 9: 400-416.

40. Zhang G, He M, Wu P, Zhang X, Zhou K, Li T, Zhang T, Xie K, Dai G, Wang J. MicroRNA-27b-3p targets the myostatin gene to regulate myoblast proliferation and is involved in myoblast differentiation. Cells. 2021; 10: 423.

41. Glass DJ. Skeletal muscle hypertrophy and atrophy signaling pathways. Int J biochem. Cell B. 2005; 37: 1974-1984.

42. Schiaffino S, Dyar KA, Ciciliot S, Blaauw B, Sandri M. Mechanisms regulating skeletal muscle growth and atrophy. FEBS J. 2013; 280: 4294-4314.

43. Liang G, Yang Y, Niu G, Tang Z, Li K. Genome-wide profiling of Sus scrofa circular RNAs across nine organs and three developmental stages. DNA Res. 2017; 24: 523-535.

44. Cao H, Liu J, Du T, Liu Y, Shi X. Circular RNA screening identifies circMYLK4 as a regulator of fast/slow myofibers in porcine skeletal muscles. 2020.

45. Dedeic Z, Cetera M, Cohen TV, Holaska JM. Emerin inhibits Lmo7 binding to the Pax3 and MyoD promoters and expression of myoblast proliferation genes. J Cell Sci. 2011;124:1691-1702.

46. Possidonio AC, Soares CP, Fontenele M, Morris ER, Mouly V, Costa ML, Mermelstein C. Knockdown of Lmo7 inhibits chick myogenesis. FEBS Lett. 2016; 590: 317-329.

47. Sun L, Ma K, Wang H, Xiao F, Gao Y, Zhang W, Wang K, Gao X, Ip N, Wu Z. JAK1-STAT1-STAT3, a key pathway promoting proliferation and preventing premature differentiation of myoblasts. J Cell Biol. 2007; 179: 129-38.

48. Begue G, Douillard A, Galbes O, Rossano B, Vernus B, Candau R, Py G. Early activation of rat skeletal muscle IL-6/STAT1/STAT3 dependent gene expression in resistance exercise linked to hypertrophy. PLoS One. 2013; 8: e57141.

49. Zhu M, Chen G, Yang Y, Yang J, Qin B, Gu L. miR-217-5p regulates myogenesis in skeletal muscle stem cells by targeting FGFR2. Mol Med Rep. 2020; 22: 850-858. 
50. Chen JF, Mandel EM, Thomson JM, Wu Q, Callis TE, Hammond SM, Conlon FL, Wang DZ. The role of microRNA-1 and microRNA-133 in skeletal muscle proliferation and differentiation. Nat Genet. 2006; 38: 228-33.

51. Dey BK, Gagan J, Yan Z, Dutta A. miR-26a is required for skeletal muscle differentiation and regeneration in mice. Gene Dev. 2012; 26: 2180-2191.

52. Ling YH, Sui MH, Zheng Q, Wang KY, Wu H, Li WY, Liu Y, Chu MX, Fang FG, Xu LN. miR-27b regulates myogenic proliferation and differentiation by targeting Pax3 in goat. Sci Rep. 2018; 8: 3909.

53. Yin H, He H, Cao X, Shen X, Han S, Cui C, Zhao J, Wei Y, Chen Y, Xia L, et al. MiR-148a-3p regulates skeletal muscle satellite cell differentiation and apoptosis via the PI3K/AKT signaling pathway by targeting MEOX2. Front Genet. 2020; 11: 512.

54. Lefebvre V, Li P, de Crombrugghe B. A new long form of Sox5 (L-Sox5), Sox6 and Sox9 are coexpressed in chondrogenesis and cooperatively activate the type II collagen gene. EMBO J. 1998; 17: $5718-5733$.

55. Kimball SR, Jefferson LS. Signaling pathways and molecular mechanisms through which branchedchain amino acids mediate translational control of protein synthesis. J Nutr. 2006; 136(1 Suppl): 227S-31S.

56. Bar DZ, Charar C, GruenbaumY. Small GTPases in C. elegans metabolism. Small GTPases. 2018; 9: 415-419.

57. Reyes NL, Banks GB, Tsang M, Margineantu D, Gu H, Djukovic D, Chan J, TorresM, Liggitt HD, Hirenallur-S DK, et al. Fnip1 regulates skeletal muscle fiber type specification, fatigue resistance, and susceptibility to muscular dystrophy. Proc Natl Acad Sci USA. 2015; 112: 424-429.

58. De Rubeis S, Pasciuto E, Li KW, Fernández E, Di Marino D, Buzzi A, Ostroff LE, Klann E, Zwartkruis FJ, Komiyama NH, et al. CYFIP1 coordinates mRNA translation and cytoskeleton remodeling to ensure proper dendritic spine formation. Neuron. 2013; 79: 1169-1182.

59. Berdeaux R, Stewart R. cAMP signaling in skeletal muscle adaptation: hypertrophy, metabolism, and regeneration. Am J Physiol Endocrinol Metab. 2012; 303: 1-17.

60. Madsen L, Kristiansen $\mathrm{K}$. The importance of dietary modulation of cAMP and insulin signaling in adipose tissue and the development of obesity. Ann NY Acad Sci. 2010; 1190: 1-14.

61. Olson EN, Spizz G, Tainsky MA. The oncogenic forms of N-ras or H-ras prevent skeletal myoblast differentiation. Mol Cell Biol. 1987; 7: 2104-2111.

62. Mitin N, Kudla AJ, Konieczny SF, Taparowsky EJ. Differential effects of Ras signaling through NFkappaB on skeletal myogenesis. Oncogene. 2001; 20: 1276-1286.

63. Murholm M, Dixen K, Hansen JB. Ras signalling regulates differentiation and UCP1 expression in models of brown adipogenesis. Biochim Biophys Acta. 2010; 1800: 619-627.

64. Kuo IY, Ehrlich BE. Signaling in muscle contraction. CSH Perspect Biol. 2015; 7: a006023.

65. Nishikimi T, lemura-Inaba C, Akimoto K, Ishikawa K, Koshikawa S, Matsuoka H. Stimulatory and Inhibitory regulation of lipolysis by the NPR-A/cGMP/PKG and NPR-C/G(i) pathways in rat cultured 
adipocytes. Regul Peptides. 2009; 153: 56-63.

66. Fadia H, Adams GR. Inhibition of MAP/ERK kinase prevents IGF-l-induced hypertrophy in rat muscles. J Appl Physiol. 2004; 96: 203.

67. Li J, Johnson SE. ERK2 is required for efficient terminal differentiation of skeletal myoblasts. Biochem Bioph Res Co. 2006; 345: 1425-1433.

68. Wei W, He HB, Zhang WY, Zhang HX, Bai JB, Liu HZ, Cao JH, Chang KC, Li XY, Zhao SH. miR-29 targets Akt3 to reduce proliferation and facilitate differentiation of myoblasts in skeletal muscle development. Cell Death Dis. 2013; 4: e668.

69. Ronzoni F, Ceccarelli G, Perini I, Benedetti L, Galli D, Mulas F, Balli M, Magenes G, Bellazzi R, et al. Metactivating genetically improved chimeric factor-1 promotes angiogenesis and hypertrophy in adult myogenesis. Curr Pharm Biotechno. 2017; 18: 309-317.

70. Cassano M, Biressi S, Finan A, Benedetti L, Omes C, Boratto R, Martin F, Allegretti M, Broccoli V, Cusella De Angelis G, et al. Magic-factor 1, a partial agonist of Met, induces muscle hypertrophy by protecting myogenic progenitors from apoptosis. PLoS One. 2008; 3: e3223.

71. Schiaffino S, Dyar KA, Calabria E. Skeletal muscle mass is controlled by the MRF4-MEF2 axis. Curr Opin Clin Nutr Metab Care. 2018; 21: 164-167.

72. Hunter RB, Kandarian SC. Disruption of either the Nfkb1 or the Bcl3 gene inhibits skeletal muscle atrophy. J Clin Invest. 2004; 114: 1504-1511.

73. Zhao X, Mo D, Li A, Gong W, Xiao S, Zhang Y, Qin L, Niu Y, Guo Y, Liu X, et al. Comparative analyses by sequencing of transcriptomes during skeletal muscle development between pig breeds differing in muscle growth rate and fatness. PLoS One. 2011; 6: e19774.

74. Ding L, Zhang L, Biswas S, Schugar RC, Brown JM, Byzova T, Podrez E. Akt3 inhibits adipogenesis and protects from diet-induced obesity via WNK1/SGK1 signaling. JCI Insight. 2017; 2: e95687.

75. Wu W, Wang S, Xu Z, Wang X, Feng J, Shan T, Wang Y. Betaine promotes lipid accumulation in adipogenic-differentiated skeletal muscle cells through ERK/PPARY signalling pathway. Mol Cell Biochem. 2018; 447: 137-149.

76. Hirata Y, Nomura K, Senga Y, Okada Y, Kobayashi K, Okamoto S, Minokoshi Y, Imamura M, Takeda S, Hosooka T, et al. Hyperglycemia induces skeletal muscle atrophy via a WWP1/KLF15 axis. JCI Insight. 2019; 4: e124952.

77. Bélanger G, Stocksley MA, Vromme M, Schaeffer L, Furic L, Desgroseillers L, Jasmin BJ. Localization of the RNA-binding proteins Staufen 1 and Staufen2 at the mammalian neuromuscular junction. $J$ Neurochem. 2003; 86: 669-677.

78. Zhang Q, Wang K, Yong Z, Meng J, Yu F, Yan C, Zhu D. The myostatin-induced E3 ubiquitin ligase RNF13 negatively regulates the proliferation of chicken myoblasts. FEBS J. 2010; 277: 466-476.

79. Li Z, Cai B, Abdalla BA, Zhu X, Zheng M, Han P, Nie Q, Zhang X. LnclRS1 controls muscle atrophy via sponging miR-15 family to activate IGF1-PI3K/AKT pathway. J Cachexia Sarcopeni. 2019; 10: 391410. 


\section{Figures}

A

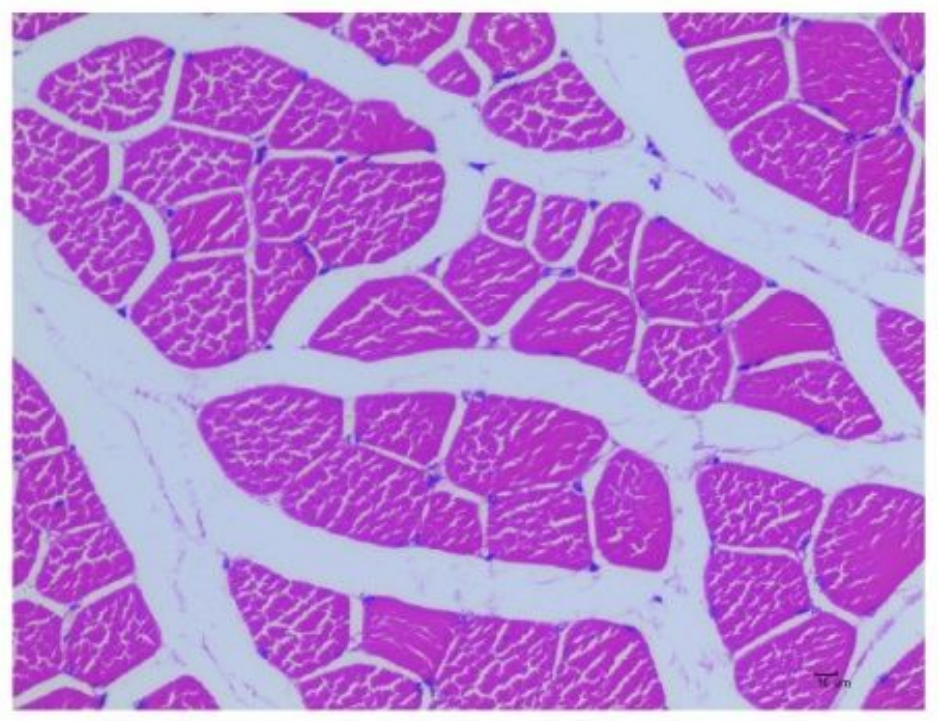

\section{LC goats}

B

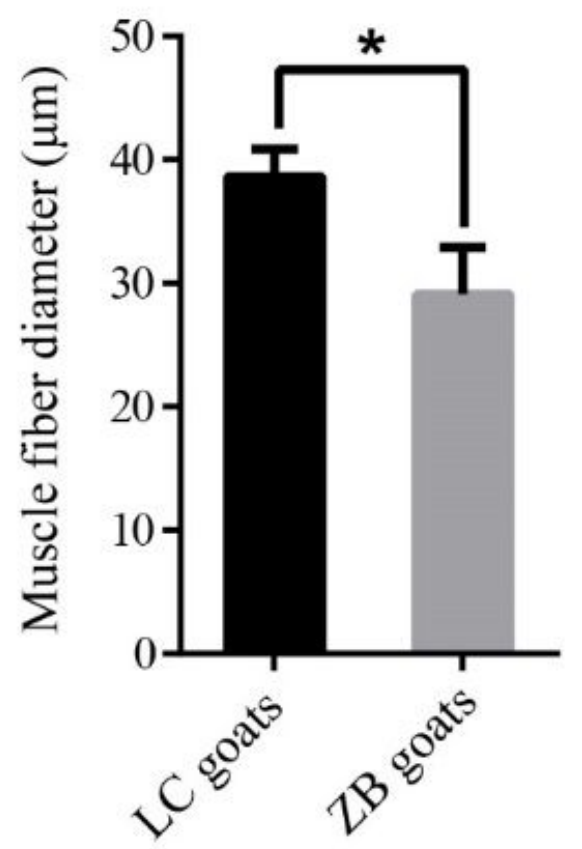

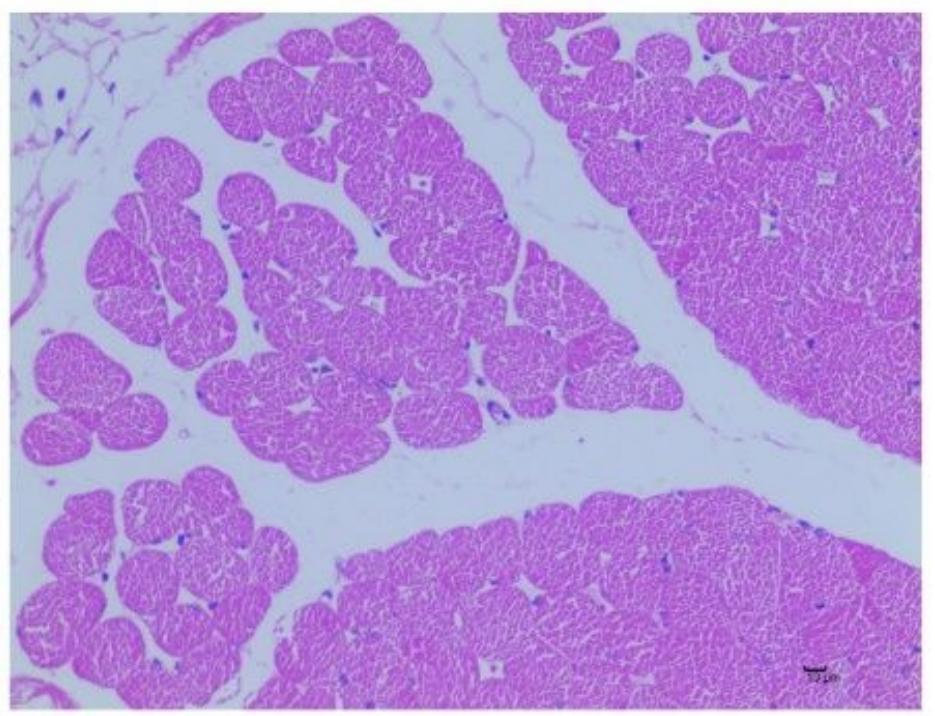

ZB goats

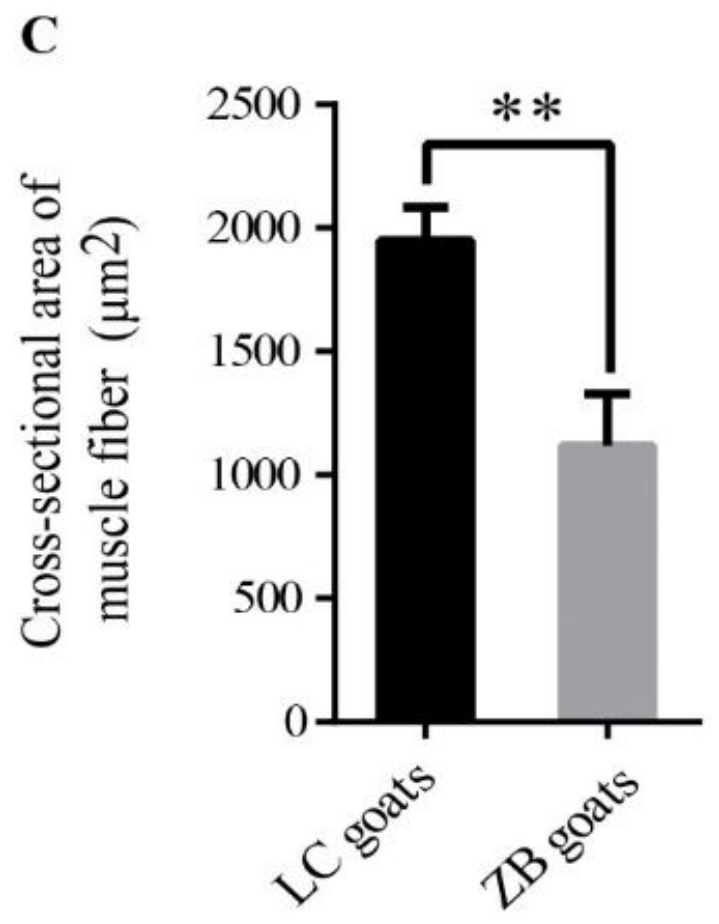

Figure 1

Comparison of the size of Longissimus dorsi muscle fiber between Liaoning cashmere (LC) goats and Ziwuling black (ZB) goats. (A) The hematoxylin and eosin staining results of Longissimus dorsi muscle tissues from LC and ZB goats. Muscle fibers were stained with red. (B) Comparison of the diameter of Longissimus dorsi muscle fiber between LC and ZB goats. (C) Comparison of the cross-sectional area of Longissimus dorsi muscle fiber between LC and ZB goats. ${ }^{*} \mathrm{P}<0.05 ; * * \mathrm{P}<0.01$. 


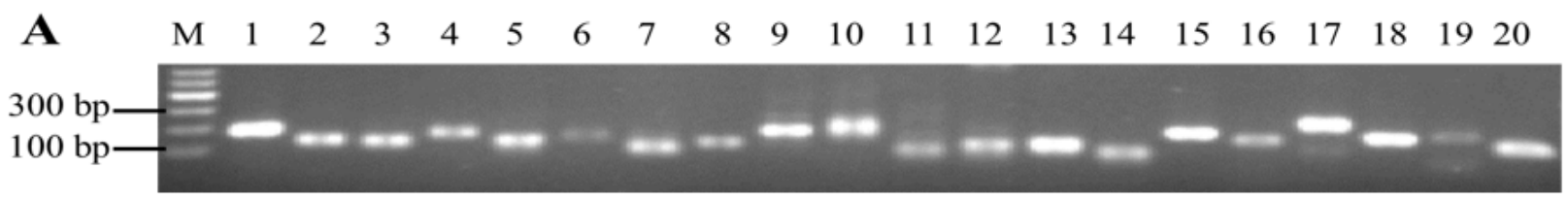

B

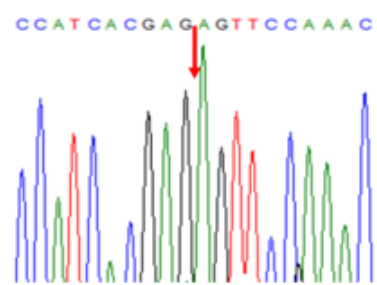

circ_009262
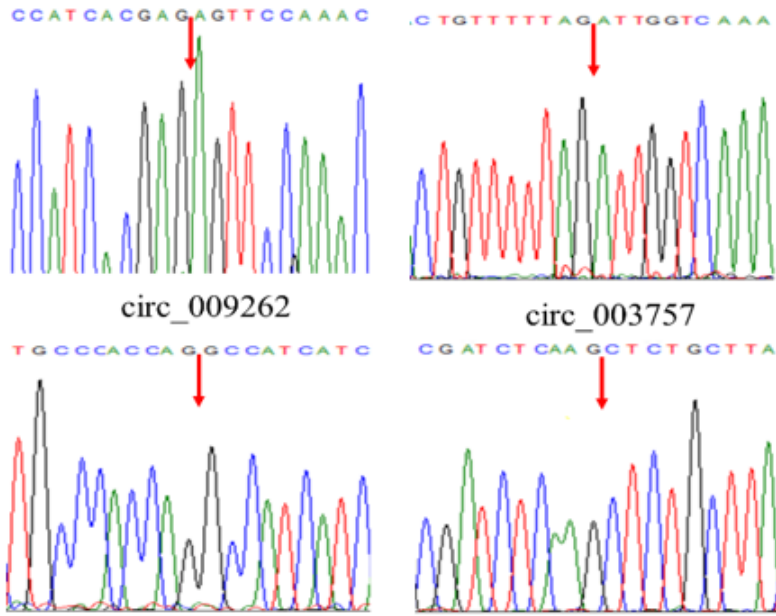

circ_003757

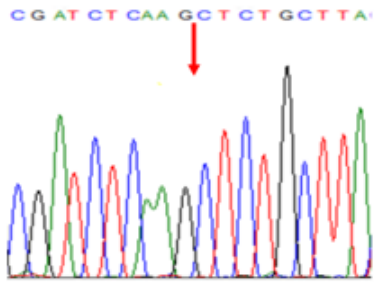

circ_007137

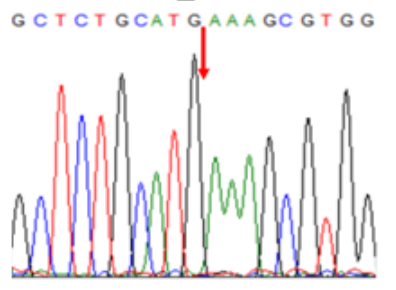

circ_008770
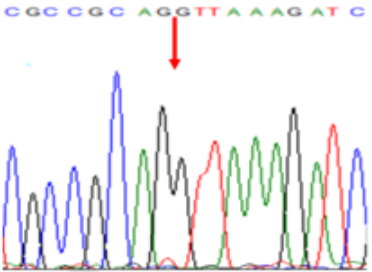

circ_004394
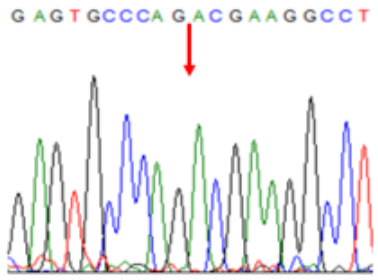

circ_010303

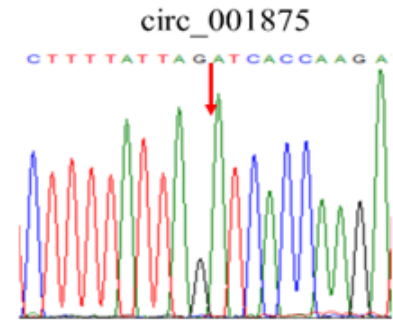

circ_009387

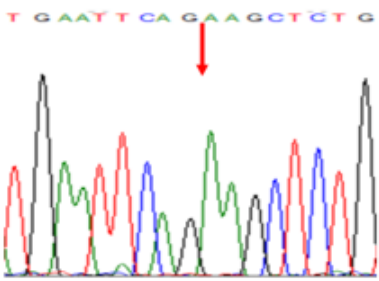

circ_003976
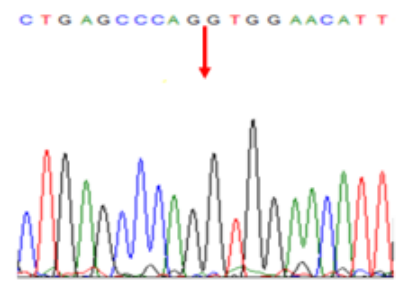

circ 007151

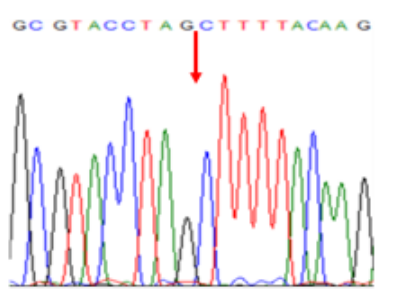

circ_008447

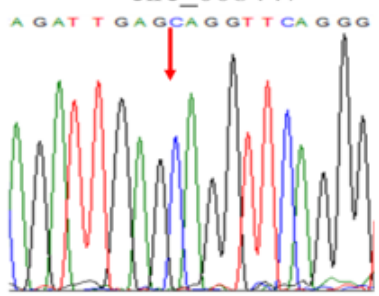

circ_006718

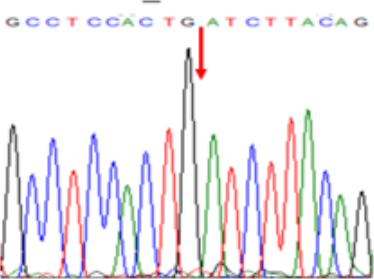

circ_006172

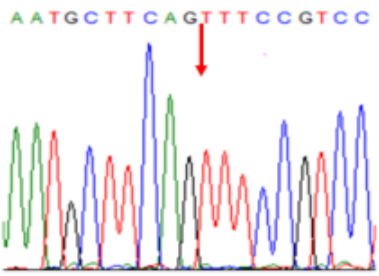

circ_002473

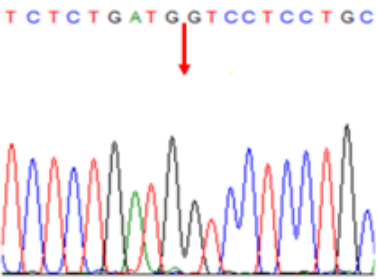

circ_008117

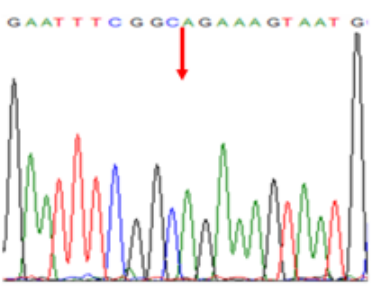

circ_002956

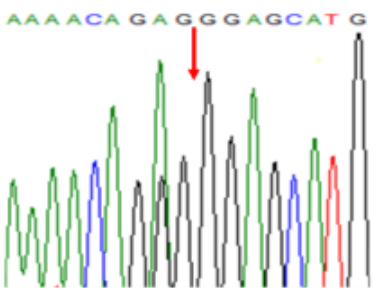

circ_008092
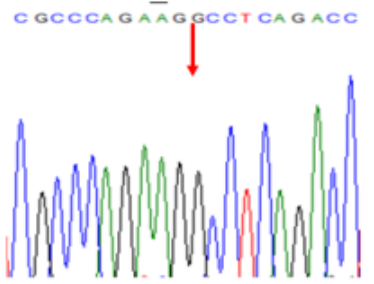

circ_005348
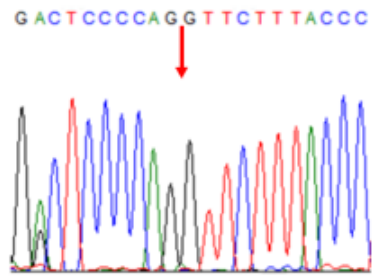

circ_008047

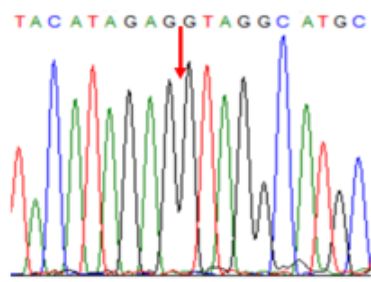

circ_009217

\section{Figure 2}

Verification of the authentication of 20 circRNAs in caprine Longissimus dorsi muscle tissues. (A) The detection of RT-PCR products of the circRNAs amplified by diver-gent primers using agarose gel electrophoresis. M: Marker; 1: circ_009262; 2: circ_003757; 3: circ_008447; 4: circ_002956; 5: circ_007137; 6: circ_001875; 7: circ_006718; 8: circ_008092; 9: circ_008770; 10: circ_009387; 11: circ_006172; 12: circ_005348; 13: circ_004394; 14: circ_003976; 15: circ_002473; 16: circ_008047; 17: circ_010303; 18 : 
circ_007151; 19: circ_008117; 20: circ_009217. (B) Validation of the back-splicing junctions for the circRNAs using Sanger sequencing. A red arrow represents the junction site on the DNA sequence chromatograms.

A

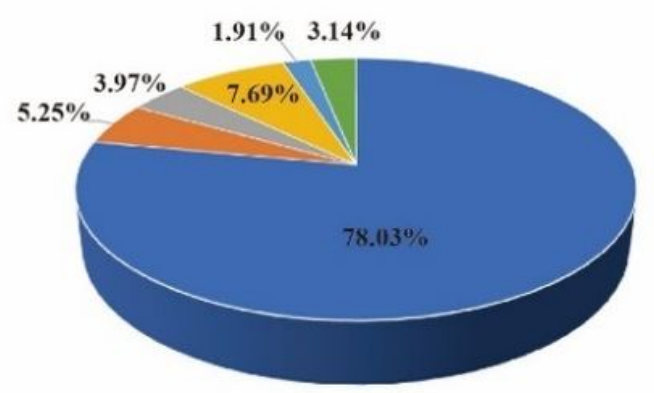

B

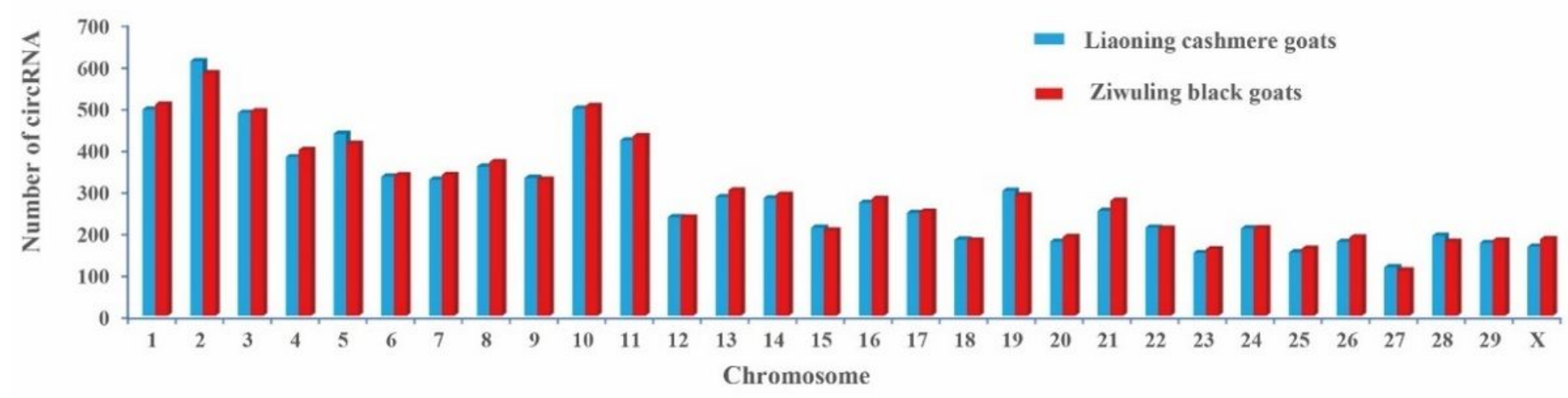

\section{Figure 3}

General characteristics of circRNAs in the caprine Longissimus dorsi muscle tissues. (A) The types of circRNAs in the caprine Longissimus dorsi muscle tissues. (B) The chromosome distribution of the sequences from which circRNAs were derived. (C) The length distribution of the circRNAs from the caprine Longissimus dorsi muscle tissues. 


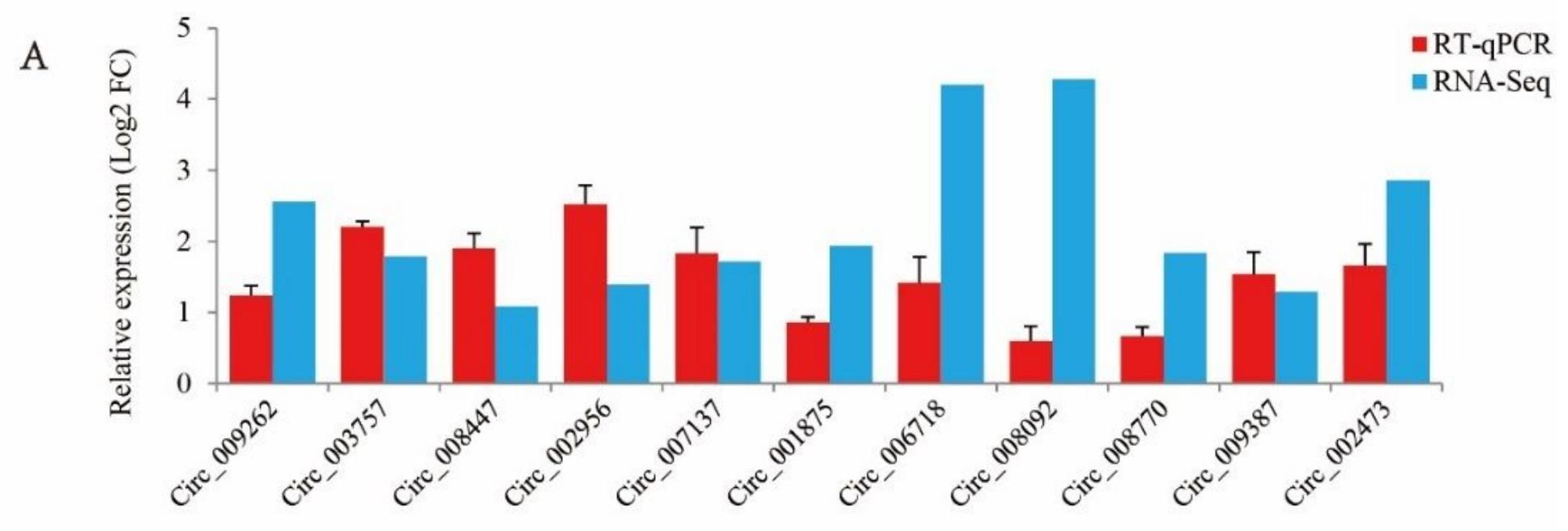

B

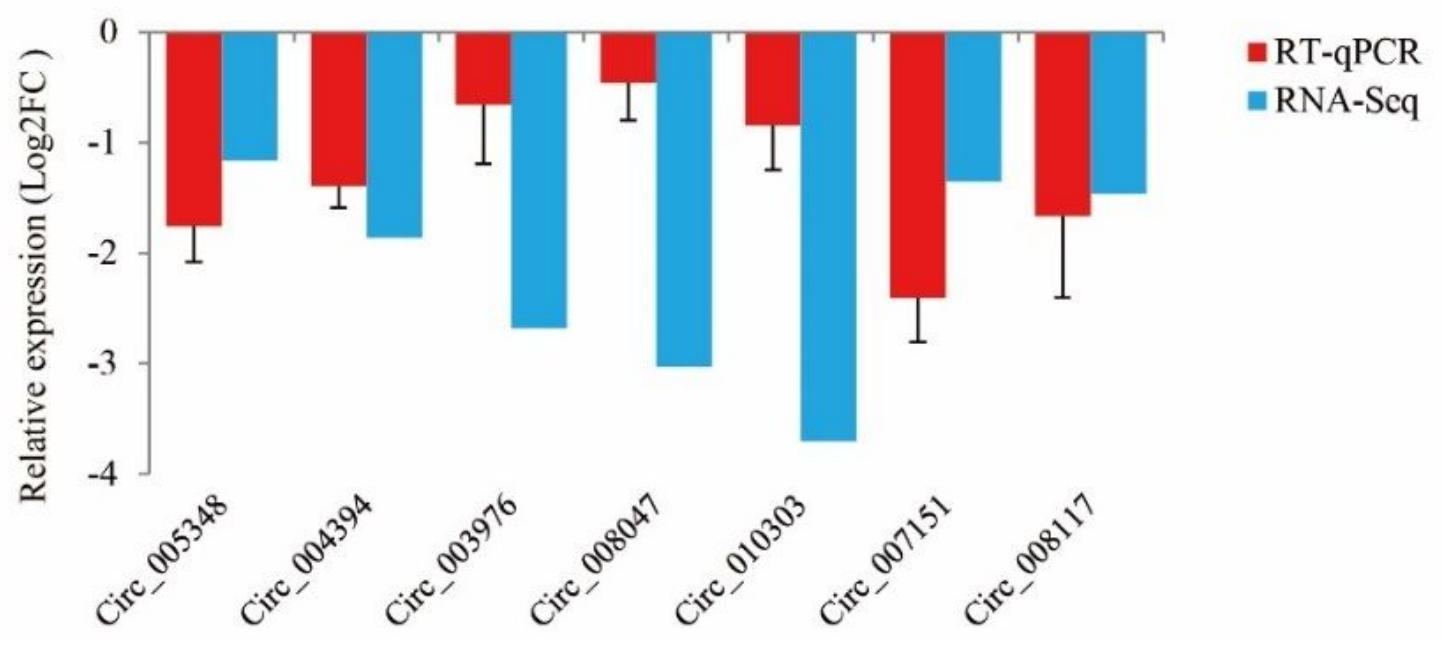

Figure 4

RT-qPCR validation of differentially expressed circRNAs identified using RNA-Seq. These included 11 upregulated circRNAs $(A)$ and 7 down-regulated circRNAs $(B)$ in LC goats compared to ZB goats. 


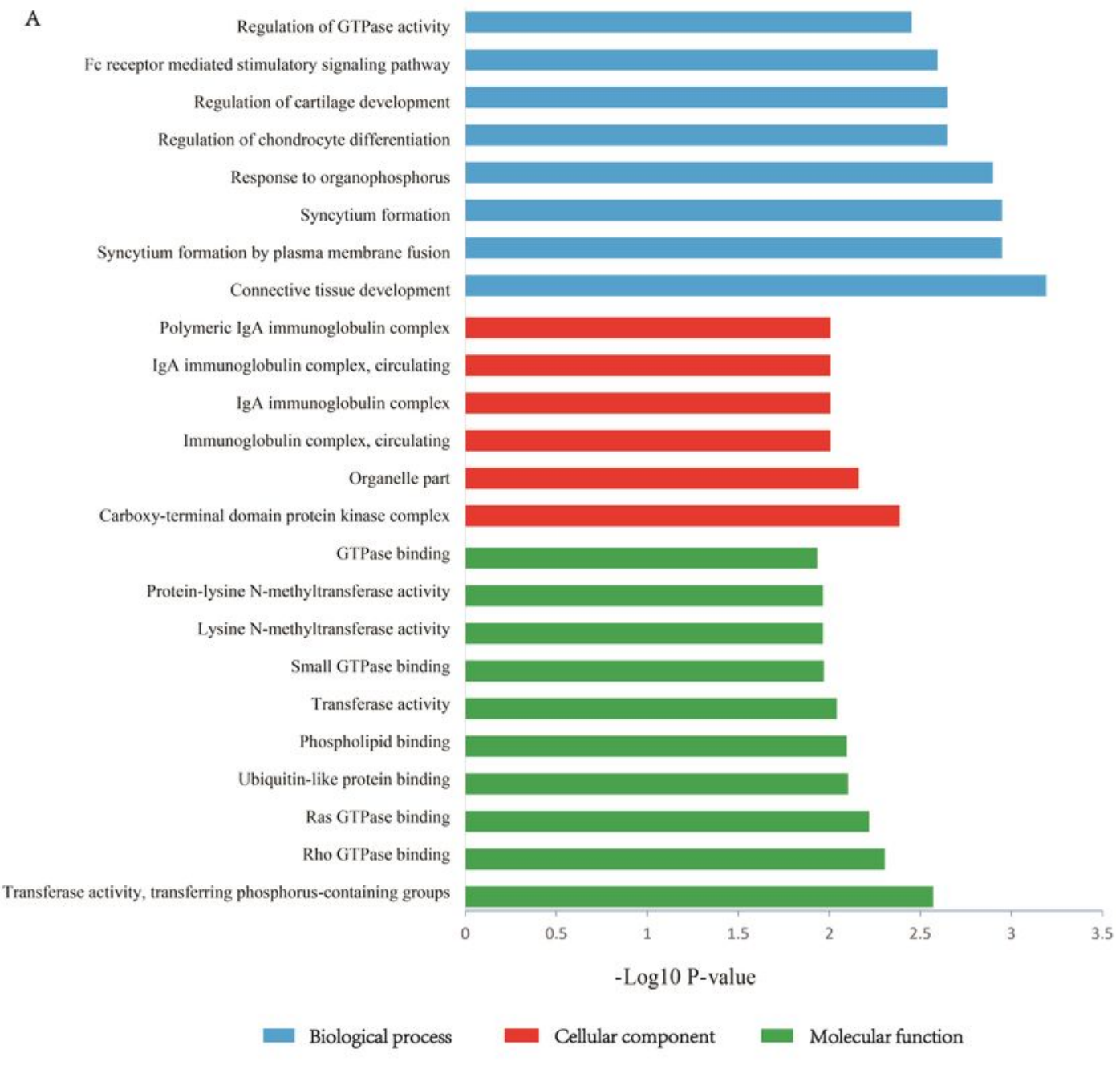

B

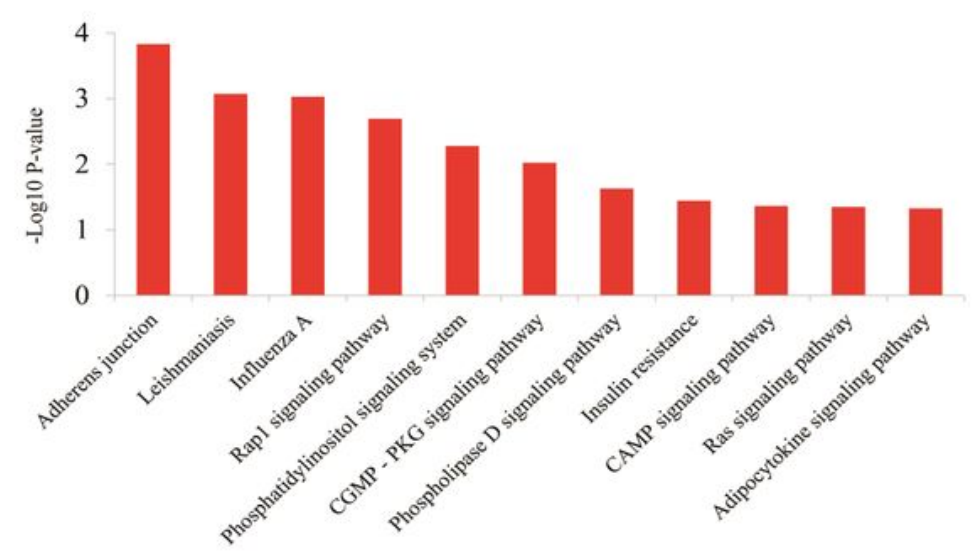

\section{Figure 5}

Function annotation of the parent genes from which the differentially ex-pressed circRNAs were derived between Liaoning cashmere (LC) goats and Ziwuling black (ZB) goats. (A) GO classification of the parent genes of the differ-entially expressed circRNAs between the two breeds. (B) KEGG enrichment analysis for the parent genes of the differentially expressed circRNAs between the two breeds. The most significant GO terms and KEGG pathways with the lowest $P$ values are shown. 


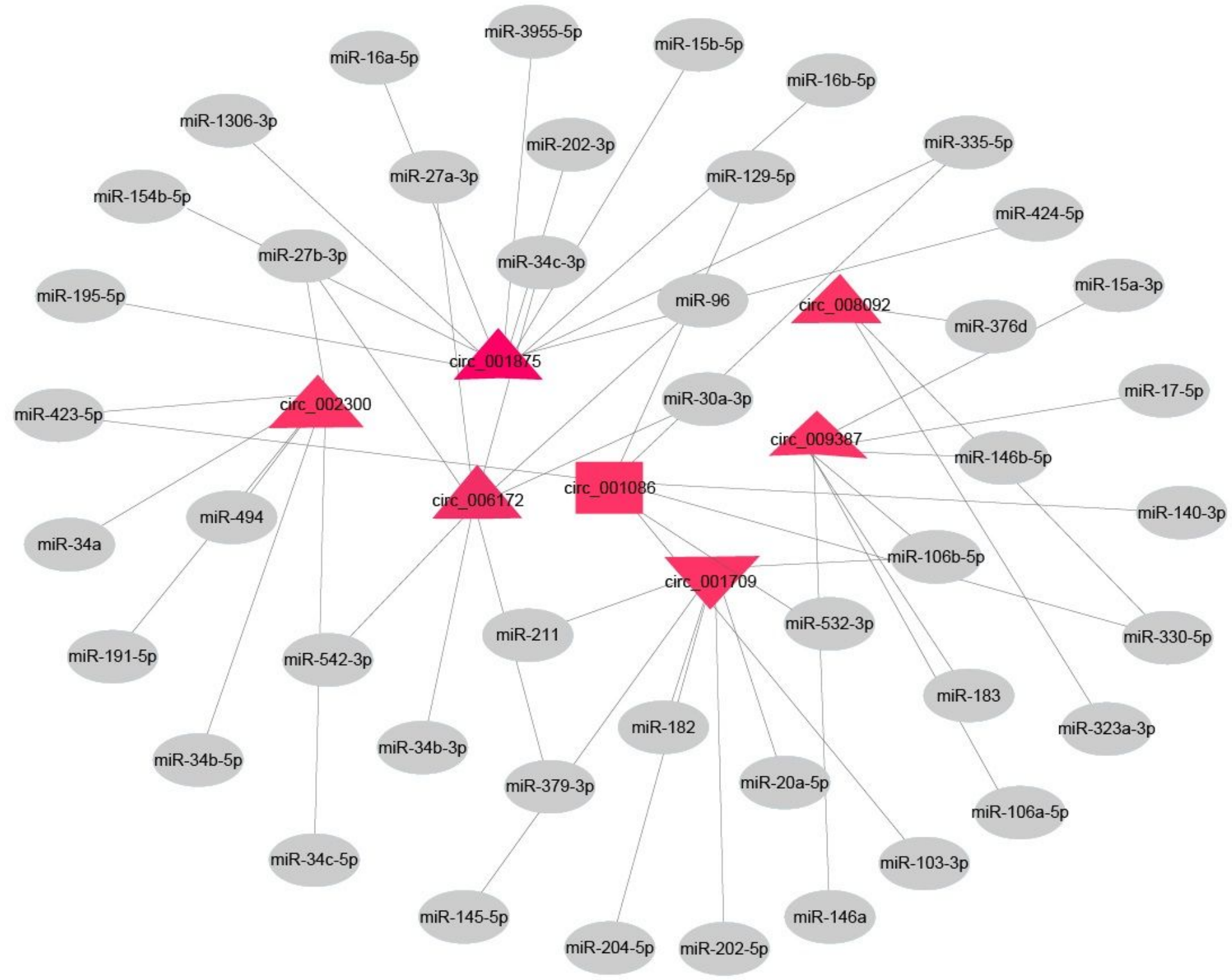

\section{Figure 6}

Interaction network of circRNA-miRNA. The red triangles and inverted triangles represent up-regulated and down-regulated circRNAs in LC goats compared to ZB goats, respectively. The red square represents the most highly expressed circRNA, while the gray circles represent the predicted target miRNAs for the 7 circRNAs.

\section{Supplementary Files}

This is a list of supplementary files associated with this preprint. Click to download.

- Supplementaryfile1.xlsx 